\title{
NICMOS3 DETECTOR FOR SPECTROSCOPY
}

\author{
L. Vanzi, A. Marconi and \\ Universita degli Studi di Firenze, Dipartimento di Astronomia \\ S. Gennari \\ Osservatorio Astrofisico di Arcetri
}

\section{INTRODUCTION}

We present some results about the use of an engineering grade NICMOS3 array for medium resolution spectroscopy. All the work described here was performed at the Infrared Laboratory of the Osservatorio Astrofisico di Arcetri in Florence and at the Italian Telescope TIRGO (Citterio 1978). The detector has been mounted on the LonGSp (Longslit Gornergrat Spectrometer), an instrument designed and set into operation in Arcetri and described in Gennari and Vanzi (1994).

This paper deals with the characterization of engineering grade arrays and with some problems in the reduction of infrared spectra.

\section{CHARACTERIZATION OF THE ARRAY}

Our spectrometer uses a subsection of an engineering grade NICMOS3 array as a detector; our purpose is to understand if this kind of array is useful for application to spectroscopy and, meanwhile, to have a good characterization to choose the best available subsection.

\subsection{Laboratory Tests}

The characterization of the array consisted of evaluating the percentage of bad pixels, the read out noise and the dark current rate.

We acquired dark and flatfield frames, the former without any illumination of the detector and the latter with the uniform illumination of an led diode inside the dewar. The dark frames were taken at three different temperatures $\left(55,65\right.$ and $\left.78^{\circ} \mathrm{K}\right)$ with different integration times (stacks of ten frames at 1, 2, 5, 10, 20,30,60,120 and 180 sec.). The flatfield frames were obtained in three different conditions: the first series after a complete reset of the array, the second and the third after a weak and a strong illumination of the detector to test for memory effects. For each series of measurements stacks of ten frames were taken with the same integration times used for dark frames.

Bad pixels were determined from normalized dark and flatfield frames by dividing each quadrant in four by four subareas and defining bad pixels as those exceeding by $4 \sigma$ the median 
value in the subarea. In all of the following analysis bad pixels were not considered and their value was substituted with the median over an $11 \times 11$ box.

The mean and the standard deviation were computed for each stack (pixel by pixel) after having renormalized the frames to get the same median value in a selected region of the first quadrant. Therefore, the averages over $16 \times 16$ subareas were taken both for pixel stack mean and standard deviation.

The readout noise was determined as the mean standard deviation of each pixel in the stacks at one and two sec and then averaging over areas of $32 \times 32$.

The dark current rate and the bias were derived by a linear fit, pixel by pixel, of the stack mean versus integration time, with the error on the mean given by the pixel standard deviation in each stack.

\subsection{Results}

We characterized two engineering grade arrays and compared the results with the science grade chip presently mounted in the large field camera ARNICA (ARcetri Near Infrared CAmera) at the TIRGO telescope (Lisi et al. 1993). Fig. 1 compares the flatfields obtained with the two engineering grade arrays.

In Table 1 the results of our measurements over the best $100 \times 100$ pixels subsection of the two engineering grade arrays and over the whole scientific array are presented.

\section{TABLE 1}

Measured characteristics of the arrays.

\begin{tabular}{llll}
\hline \hline & $\begin{array}{l}1 \text { best } \\
100 \times 100\end{array}$ & $\begin{array}{l}2 \text { best } \\
100 \times 100\end{array}$ & Scientific \\
\hline Bad Pix (\%) & 003.0 & 01.7 & 01.0 \\
Read Out Noise (e) & 240 & 45 & 45 \\
Dark Current ( $\left.\mathrm{e}^{-} / \mathrm{s}\right)$ & 040 & 02 & 01 \\
\hline
\end{tabular}

In Fig. 2, the dark rate is plotted as a function of temperature. The three different symbols at $78^{\circ} \mathrm{K}$ indicate frames taken after complete reset (circle), after normal illumination (triangle, like measurements at lower T) and after strong illumination (squares). In the latter case one can notice an increase of the dark current rate by about $20 \%$. Indicative values for the dark rate are $0.1 \mathrm{ADU} / \mathrm{s}\left(2 \mathrm{e}^{-} / \mathrm{s}\right)$. 

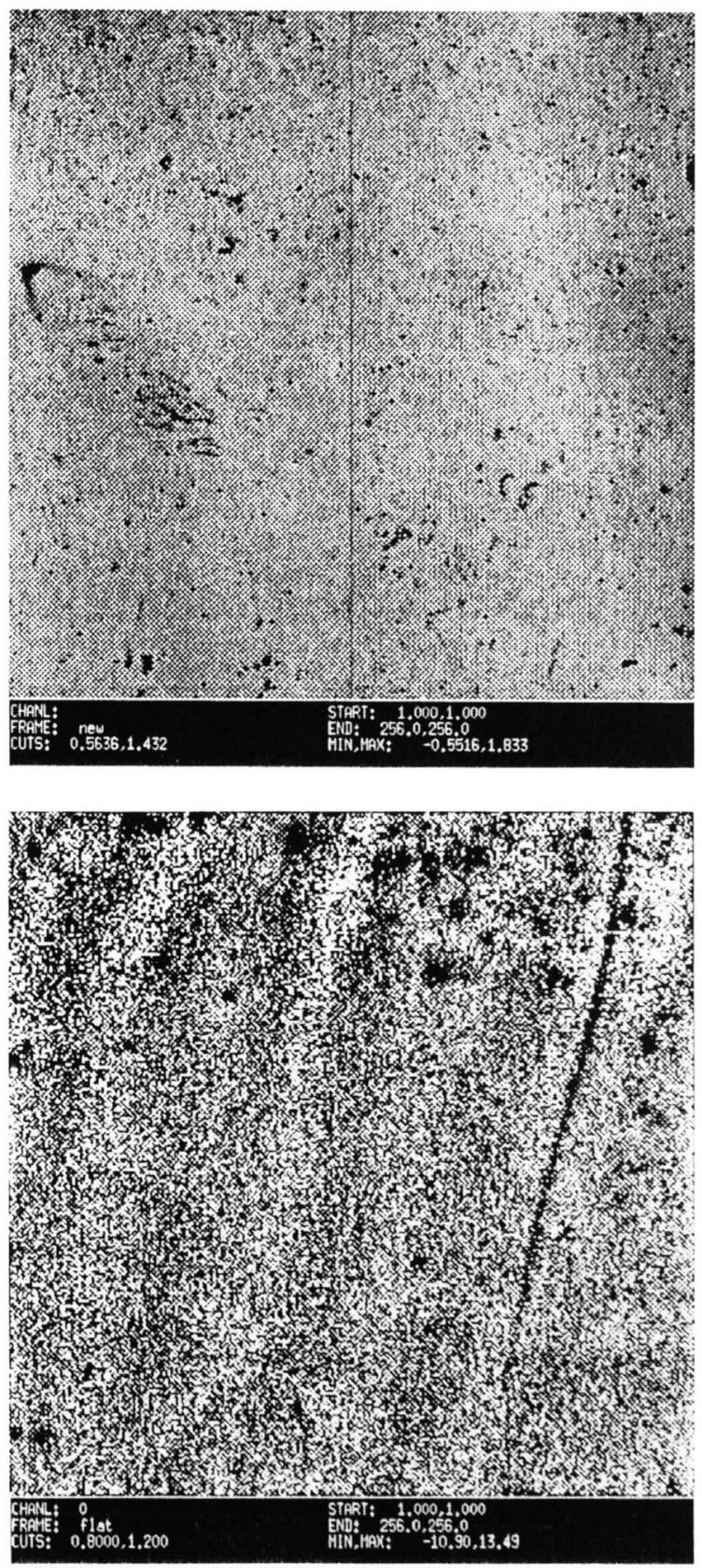

Fig. 1. Flatfield frames of the engineering grade arrays (the one now mounted on LonGSp is in the lower panel). 


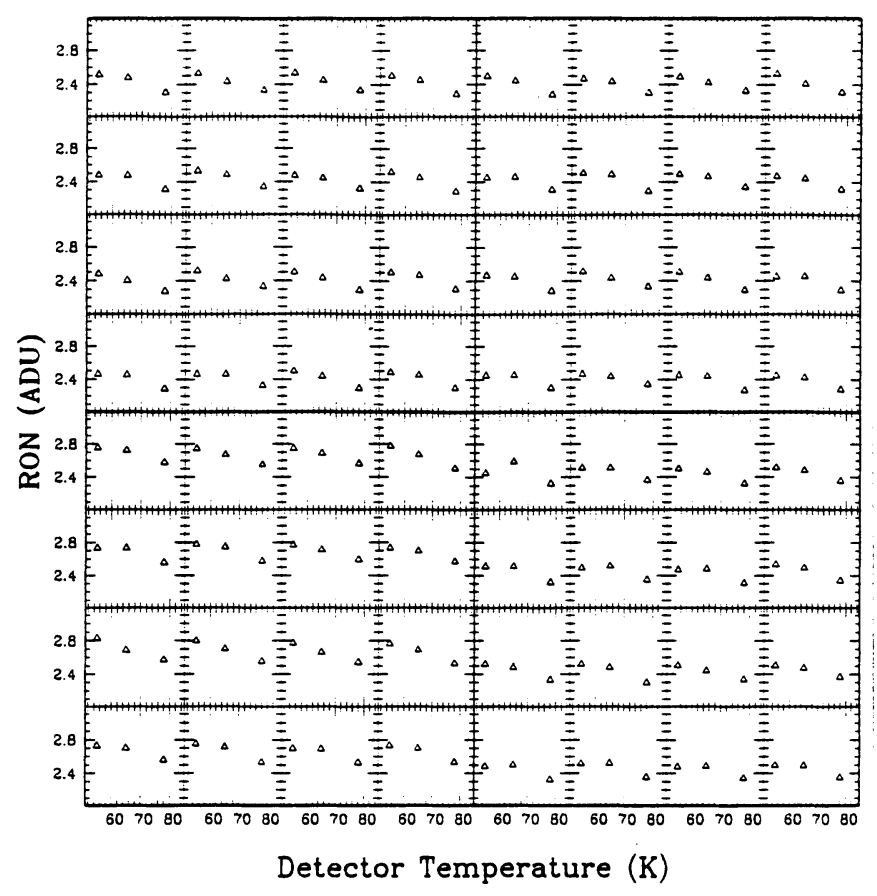

Fig. 2. Dark rate (ADU/sec) of the new engineering grade array as a function of the detector temperature and in three different conditions: after a full reset (circles), after normal (triangles) and strong illumination (squares). 


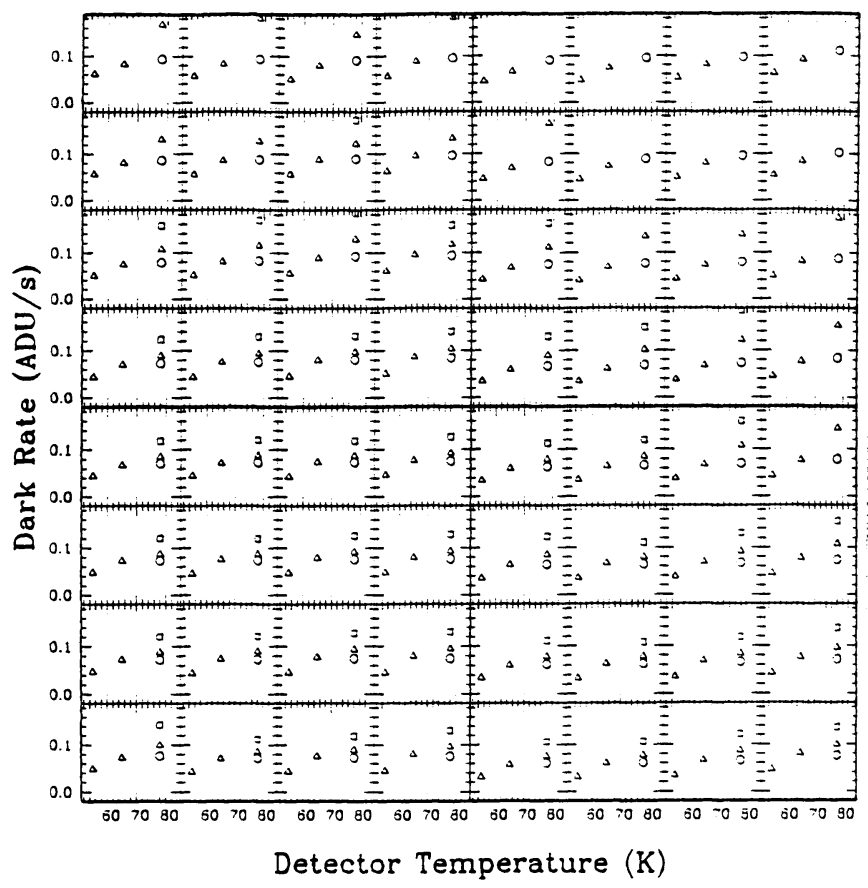

Fig. 3. Dark rate (ADU/sec) of the new engineering grade array as a function of the detector temperature and in three different conditions: after a full reset (circles), after normal (triangles) and strong illumination (squares). 


\section{DATA REDUCTION}

The data reduction is performed with the ESO package MIDAS in the context IRSPEC. The context has been optimized for reduction of IRSPEC data but can be used for LONGSP since the principles of data reduction are quite general and not specific to a particular instrument.

\subsection{Data from Observations}

Each observation includes object and sky frames with the same integration time taken with the ABBA sequence as well as the spectrum of a standard star and, if necessary, a wavelength calibration lamp. Flatfield and dark frames should be taken, for instance, at the beginning or at the end of the night or every few hours according to the stability of the detector.

\subsection{Correction for Bad Pixels}

This correction can be performed once a mask of bad pixels is given. Bad pixels are substituted by the average value over the neighboring pixel in the $\mathrm{x}$ or $\mathrm{y}$ direction. The chosen direction is that with the lower gradient. This method works only with fairly isolated bad pixels and cannot handle cases when the bad pixels are clustered. In such a situation one may use techniques similar to those for cosmic rays removal in CCD frames.

\subsection{Flatfield and Dark Current}

The original flatframe cleaned by bad pixels is divided by the average of those rows where there will be the object spectrum. After the normalization, all of those pixels that are lower than a threshold (for distance 0.5 ) are considered vignetted and set to a fixed high value (i.e. 100) so that after flat division the same pixels in the object frame will not be considered. The flat image is usually a measurement of the halogen lamp with counts level as close as possible - and well within a factor of two - to those in the astronomical frames.

\subsection{Sky Subtraction}

The greatest problem in NIR observations is the subtraction of the $\mathrm{OH}$ sky lines which are very bright and variable on time scale of a minute. A simple object-sky subtraction is not always enough to guarantee a complete removal of the emission lines as the sky spectra in the two frames (source and sky) may be different, because of a shift in the wavelength direction due to mechanical flexures in the instrument and of variation of line intensity.

The technique used is to consider the difference between object and sky frames and choose an area of the frame where there are bad subtracted sky lines. The extra noise there is mainly due to residuals of $\mathrm{OH}$ lines. The factor to rescale the sky frame and the shift to apply are fixed by minimizing the noise in the previously chosen area. In the spectral region where the background is not too high one also need a dark frame. The procedure can be summarized in the following equation:

$$
\operatorname{SKYSUB}(x, y)=\frac{O B J(x+\delta, y)-\alpha S K Y(x, y)-(1-\alpha) D A R K(x, y)}{F L A T(x, y)-D A R K(x, y)} \quad 1
$$


where $\alpha$ is the factor by which the $\mathrm{OH}$ line varied (and this correction must not be applied to the dark frame!) and $\delta$ is the shift due to grating movements. $\alpha$ and $\delta$ are determined by minimizing the noise on the background.

This procedure is not able to correct grating shifts $\geq 0.1$ pixels during the integration, in that case the problem is still open. This procedure of sky subtraction becomes less and less efficient as the integration time increases, in particular one can notice how the noise gets greater than simple fluctuations of the background when integrating longer than $200 \mathrm{sec}$ (Fig. 3.)

This problem limits the integration time and increases the limiting flux (about $10^{-13} \mathrm{erg} \mathrm{s}^{-1}$ $\mathrm{cm}^{-2}$ arcsec $\mathrm{Cm}^{-1}$, preventing us from reaching background limited performances in the $\mathrm{J}$ and $\mathrm{K}$ bands.

\subsection{Rectification of the Frames}

The slit images at the various wavelengths ("spectral lines") are tilted as a consequence of the off-axis mount of the grating, and the angle by which they are tilted varies with the position of the grating, i.e. with the wavelength. The tilt angle is computed analytically from the instrumental calibration parameters (on line central wavelength etc.) so the correction does not present particular problems.

\subsection{Wavelength Calibration}

The wavelength dispersion on the array is linear within a small and totally negligible fraction of the pixel size. Hence, wavelength calibrating simply means modifying the $\mathrm{x}$-start and $\mathrm{x}$-step values (descriptor) of the image. Another advantage is that one can very precisely compute (analytically) the pixel size - in wavelength - once the central wavelength of the frame is known. A quite precise, usually within one pixel, estimate of this quantity is available on-line at the instrument ("mechanical" calibration). One can directly use this information and determine the "mechanical" wavelength calibration. It is possible to determine more precisely the central wavelength of the frame, and hence to obtain a very accurate wavelength calibration, if one has a frame containing lines with known wavelengths; up to 2.3 microns the $\mathrm{OH}$ lines in the sky frames are a very convenient calibrator (Oliva and Origlia 1992).

\subsection{Calibration with a Standard Star}

The calibration procedures up to wavelength calibration followed for the object must be repeated also for a standard star. Having flux calibrated the spectrum of a standard star with known photometric data one divides the object frame by the standard star, pixel by pixel, to achieve the cancellation of atmospheric absorption lines and flux calibrate the spectrum. Possibly there might be small shifts and different widths between absorption features in object and star spectra. In that case one determines by hand the shift and the smoothing to apply to the star to better remove the features. 


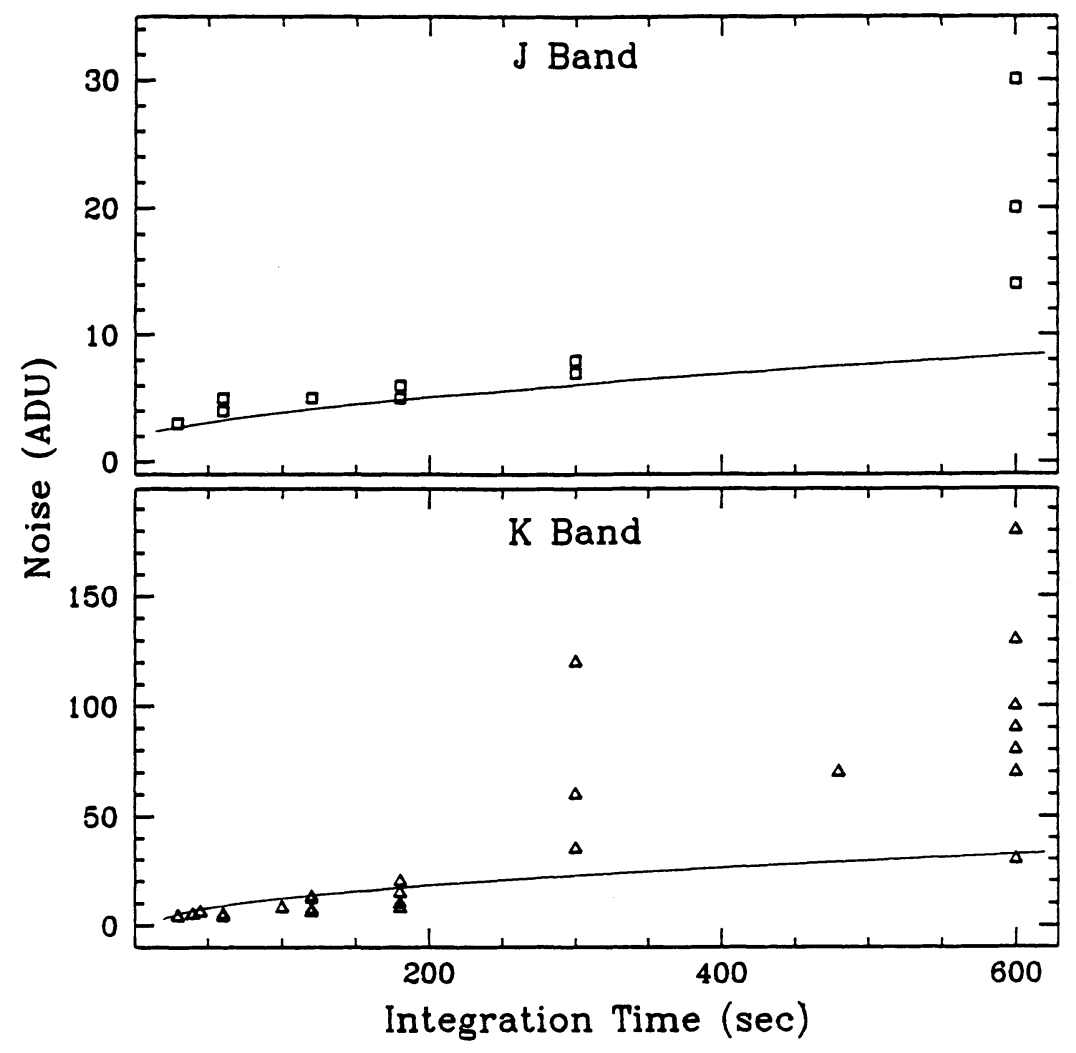

Fig. 4. Noise (ADU) of the sky subtracted images as a function of the integration time. The lines represent the Poissonian component of the background noise: note the high noise due to bad subtraction of $\mathrm{OH}$ sky lines after $200 \mathrm{sec}$ of on-chip integration. 


\section{CONCLUSION}

Engineering grade arrays have quite large subsections with performances comparable to a scientific grade array and can be a cheap solution if a large field is not required. Doing infrared spectroscopy at medium resolution with these arrays allows the use of quite long integration times and the limits on the signal to noise ratio are mainly posed by the subtraction of sky lines.

\section{REFERENCES}

Citterio, X 1978 Mem. Soc. Astron. It. 49, 57

Gennari S. and Vanzi L. 1994 in Experimental Astronomy 3, 191, InfraRed Astronomy with Arrays, I. McLean, ed.

Lisi, F., Baffa, C. and Hunt, L. K. 1993 Communication 1946.58 at the International Symposium of SPIE, The international Society for Optical Engineering, Orlando (USA) 12-16 April 1993

Oliva, E. and Origlia, L. 1992 A\&A 254, 466

\section{DISCUSSION}

CORBALLY: Are you aware of any significant differences between your Nicmos-based spectrometer and a similarly based one of George Rieke?

VANZI: I know George Rieke has a similar spectrometer but actually I am not aware if he has similar problems. I know about other spectrometers; it seems they don't have problems of sky subtraction but all use much shorter integration times.

CUBY: Can you comment on persistence effects which may be important after calibrations (flatfields or standard stars) at high flux levels.

VANZI: Yes, they are. What we have observed is that after a strong illumination of the detector you have to reset it many times to remove completely any persistence effect. This depends on how strong the illumination was. 